\title{
EXISTENCE OF SOLUTIONS OF DIFFERENTIAL EQUATIONS IN BANACH SPACE
}

\author{
BY WILLIAM J. KNIGHT
}

Communicated by Fred Brauer, July 23, 1973

The results announced here concern the existence of a solution to the general initial value problem

$$
x^{\prime}(t)=f(t, x(t)), \quad x(0)=x_{0},
$$

in which $x(t)$ lies in a Banach space $X$ for $t \in J=[0, a]$. Recent results for this problem have been announced in this Bulletin by S. N. Chow and J. D. Schuur [1] and by W. E. Fitzgibbon [2]. Related results were obtained earlier by F. Browder [3]. Here however, $X$ is not assumed to be separable or reflexive, although as usual $f$ will be continuous in $x$ with respect to the weak topology on $X$.

A pseudo-solution of (1) is an absolutely continuous function $x: J \rightarrow X$ with pseudo-derivative (see Pettis [4]) satisfying (1). A strong solution of (1) is a strongly absolutely continuous function $x: J \rightarrow X$ with strong derivative $\left(\lim _{h \rightarrow 0}(x(t+h)-x(t)) / h\right.$ in norm) satisfying (1) a.e. on $J$. For notions of absolute continuity, see Hille and Phillips [5, p. 76].

In what follows let $B$ denote an open ball about some point $x_{0} \in X$, let $I=[0, b]$ be a compact interval, and let $f$ be a function from $I \times B$ into $X$.

Theorem A. Assume these hypotheses:

(a) For a.e. $t \in I, f(t, x)$ is continuous in the variable $x$ with respect to the weak topology on $B$ and $X$.

(b) For each strongly absolutely continuous function $y: I \rightarrow B, f(t, y(t))$ is Pettis integrable on I.

(c) For some null set $N \subset I$, the weak closure of $f((I-N) \times B)$ is weakly compact in $X$.

Then (1) has a (possibly nonunique) pseudo-solution on a subinterval $J=[0, a]$ of $I$.

AMS (MOS) subject classifications (1970). Primary 34G05; Secondary 47H10.

Key words and phrases. Initial value problem, pseudo-solution, pseudo-derivative, strong solution, fixed point, strongly measurable, uniformly convex space. 
The proof of Theorem A applies the Schauder-Tychonoff fixed point theorem to the transformation $T$ defined by

$$
T y(t)=x_{0}+\int_{0}^{t} f(s, y(s)) d s \quad \text { (Pettis integral) }
$$

on the intersection of certain convex subsets of the locally convex product space $X^{J}$ of all functions from $J$ into $X$.

Sufficient conditions for (b) to hold are that $X$ be weakly sequentially complete, that $f(t, y(t))$ be weakly measurable for each strongly absolutely continuous function $y: I \rightarrow B$, and that (c) hold. If we require that $f(t, y(t))$ be strongly measurable, we obtain the existence of a strong solution.

Corollary B. In Theorem A, replace condition (b) by the following condition.

$$
\begin{aligned}
& \text { For every strongly absolutely continuous function } \\
& y: I \rightarrow B, f(t, y(t)) \text { is strongly measurable on } I .
\end{aligned}
$$

(This condition, together with (c), implies (b).)

Then every pseudo-solution of (1) is in fact a strong solution.

A simple sufficient condition for $\left(b^{*}\right)$ to hold is that for each point $x \in B, f(t, x)$ be strongly measurable with respect to $t$ on $I$.

Strong solutions of (1) will also be obtained in Theorem A if $X$ is uniformly convex, for pseudo-solutions of (1) under hypothesis (c) are in fact strongly absolutely continuous, hence strongly differentiable by Clarkson [6].

\section{BIBLIOGRAPHY}

1. S. N. Chow and J. D. Schuur, An existence theorem for ordinary differential equations in Banach spaces, Bull. Amer. Math. Soc. 77 (1971), 1018-1020. MR 44 \#4334.

2. W. E. Fitzgibbon, Weakly continuous accretive operators, Bull. Amer. Math. Soc. 79 (1973), 473-474.

3. Felix E. Browder, Non-linear equations of evolution, Ann. of Math. (2) 80 (1964), 485-523. MR 30 \#4167.

4. B. J. Pettis, On integration in vector spaces, Trans. Amer. Math. Soc. 44 (1938), 277-304.

5. E. Hille and R. Phillips, Functional analysis and semi-groups, Amer. Math. Soc. Collog. Publ., vol. 31, Amer. Math. Soc., Providence, R.I., 1957. MR 19, 664.

6. J. A. Clarkson, Uniformly convex spaces, Trans. Amer. Math. Soc. 40 (1936), 396-414.

Department of Mathematics, Pennsylvania State University, University Park, Pennsylvania 16802 